
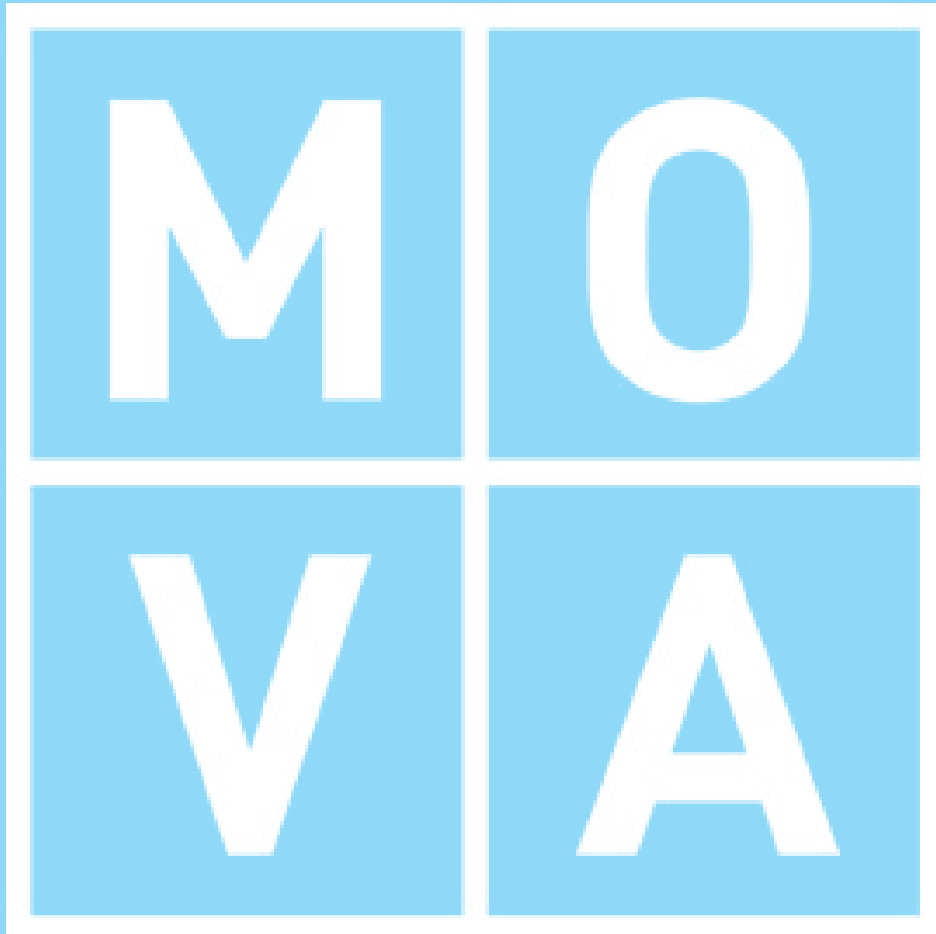

\title{
El diseño de "Mova, Centro de Innovación del Maestro": Un ejercicio colectivo desde los saberes de los maestros, las maestras y otros agentes educativos
}

Designing "Mova, Center of Innovation for the Teacher": A collective exercise of the acquired knowledge of teachers and other educational agents

Mova, Centro de Inovação do Professor: Um exercício coletivo a partir do conhecimento dos professores e outros profissionais

Lina Marcela Quintana Marín / Lía del Carmen Córdoba Garrido / Jorge Manuel Escobar Ortiz 
Lina Marcela Quintana Marín ${ }^{1}$

Lía del Carmen Córdoba Garrido² Jorge Manuel Escobar Ortiz

1 Docente en Comisión de la Secretaría de Educación de Medellín. Integrante del Grupo Historia de la Práctica Pedagógica en Colombia (GHPPC). Magister en Educación de la Universidad de Antioquia. Correo electrónico: linamarquintanana@gmail.com

2 Docente en Comisión de la Secretaría de Educación de Medellín. Estudiante de Maestría en Didáctica y Conciencia Histórica en el Instituto de Pensamiento y Cultura de América Latina (IPECAL). Correo electrónico: liacordoba@gmail.com

3 Miembro del equipo de contenidos educativos de Mova. Integrante del grupo Ciencia, Tecnología y Sociedad más Innovación (CTS+I) del Instituto Tecnológico Metropolitano de Medellín. Magíster en Filosofía (University of Manitoba) y en Historia y Filosofía de la Ciencia (University of Notre Dame). Correo electrónico: jormanescor@gmail.com

Fecha de recepción: 16 de marzo de 2015 / Fecha de aprobación: 3 de abril de 2015.

\section{Resumen}

El presente texto describe el papel de maestros, maestras y otros profesionales en la construcción de "Mova, Centro de Innovación del Maestro". Interesa destacar en particular la forma en que el saber de maestros y maestras recorrió el diseño arquitectónico y contribuyó a la configuración de líneas de formación que se expresaron en expectativas, necesidades, tensiones y soluciones para continuar con su formación. Maestros y maestras ofrecieron su experiencia, prácticas pedagógicas y sentido crítico y colectivo para generar diálogos reflexivos, tanto con colegas, como con otros profesionales y agentes educativos, sobre los distintos aspectos de Mova, incluida la construcción de los lineamientos de política de formación en servicio de la ciudad.

El proceso de construcción de Mova unió conceptos, imágenes y experiencias que fueron agrupando nuevos conocimientos, y reconociendo otros guardados en la memoria, acerca de la formación continua de los maestros de Medellín.

\section{Palabras clave}

Innovación educativa, experiencias significativas, participación docente en la construcción de saberes, práctica pedagógica, creación pedagógica.

\section{Summary}

The purpose of this article is to describe the role played by teachers and other educative actors in the design of Mova, Teachers' Center of Innovation. We want to highlight the relevance of teachers' ideas and opinions in the architectural design and the configuration of the educative strategies of Mova, which were expressed as expectations, necessities, tensions and solutions that helped in the conformation of a proposal for teacher education. Teachers offered their experience, pedagogic practices and critical sense to generate reflexive environments with colleagues and other professionals, focusing on multiple aspects about Mova, including the construction of public policy for in-service education for Medellín. This process grouped multiple concepts, images and experiences organized under new knowledge which recognized other ideas stored in the collective memory of Medellín's teachers.

\section{Key words:}

Innovation for education, significative experiences, teachers participation in the construction of knowledge, pedagogical practice, pedagogical creation.

\section{Resumo}

Este artigo descreve o papel dos professores, professoras e outros profissionais na construção de Mova, Centro de Inovação do Professor. Digno de nota, em particular, a forma como o conhecimento dos professores e professoras percorreu o conjunto arquitetônico e contribuiu para as linhas de configuração formação que expressaram expectativas, necessidades, tensões e soluções para continuar com sua educação.

Professores e professoras ofereceu sua experiência, práticas pedagógicas e senso crítico e coletiva para gerar diálogos reflexivos com colegas e outros profissionais sobre diversos aspectos da Mova, incluindo a construção das orientações políticas para a formação em serviço de Medellín.

Esse processo de construção do Mova juntou conceitos, imagens e experiências que foram recolhendo novos conhecimentos e reconhecendo outros armazenados na memória sobre a formação continuada dos professores em Medellín.

\section{Palavras chave:}

Inovacão educacional, experiências significativas, participação docente na construção de saberes, experiências significativas, prática pedagógica, criação docente. 


\section{Introducción}

$\mathrm{M}$

ova es un proyecto de la Alcaldía de Medellín que se ejecuta, desde el año 2014, por medio de la Secretaría de Educación con recursos del Fondo Medellín, Ciudad para la Vida, en convenio de asociación con el Parque Explora y con Proantioquia, como aliados estratégicos. El proyecto se encuentra aún en fase de desarrollo y nace a partir del interés de la administración municipal por la educación y el reconocimiento a maestros y maestras como ejes transformadores de los procesos educativos de la ciudad.

Mova se inscribe en el propósito, que la ciudad ha mantenido durante los últimos diez años, de incluir a la educación en la agenda pública como uno de sus elementos fundamentales. Con ello se han generado nuevas políticas y propuestas educativas, artísticas, sociales, científicas, académicas y urbanísticas que muestran a Medellín como una ciudad para la vida. Mova entra a jugar un papel preponderante en la dinamización de los espacios culturales de esta Ciudad Escuela y se integra a la idea de una ciudad que se aproxima a sus realidades en clave educativa.

Mova se ha definido como un escenario que propicia, promueve e integra las dimensiones del ser, el saber y el crear de los maestros y las maestras; a su vez, posibilita hábitos personales y profesionales encaminados a la generación de experiencias educativas y prácticas pedagógicas diversas y contextualizadas. También se comprende como una plataforma para la gestión de propuestas y acciones, generadas desde y para la comunidad docente y otros agentes educativos, con aplicabilidad y pertinencia en diversos contextos.

En la construcción de Mova se generaron espacios para la pregunta, el debate, la discusión, la confrontación, la argumentación y el análisis de situaciones educativas desde diferentes perspectivas y con diferentes actores. En este escenario se hizo posible la integración de saberes y de conocimientos que permitieron la configuración de posturas críticas frente a las concepciones de espacios de formación, de estrategias y metodologías de enseñanza, de lecturas múltiples del adentro y el afuera de la escuela. En suma, de apuestas alternativas frente a la formación de maestros y maestras en servicio.

Las conversaciones se permearon por la práctica pedagógica, bajo la defensa de la premisa de que no se pueden identificar, pensar y resolver los problemas de la educación, la formación y la enseñanza a espaldas del saber recogido por los maestros y las maestras de la ciudad. Los diálogos con los maestros y las maestras se realizaron por medio de diferentes estrategias que recogieron expectativas, tensiones, experiencias y problemáticas sobre la formación pedagógica, la cotidianidad de la escuela, la relación con los espacios y los contextos escolares y la pregunta por la investigación, entre otras. A continuación se mencionan algunas de ellas:

- Se realizaron 3 talleres de imaginarios para activar la relación pedagógica con los espacios del Centro.

- Se llevaron a cabo 27 encuentros de Rutas Narrativas de Ciudad con diferentes instituciones educativas.

- 34 encuentros con la Ruta Mova-Explora, que permitió reconocer diversos escenarios educativos de la ciudad.

- Hubo 6 campamentos pedagógicos que ayudaron a identificar y reconocer los principios de Mova por medio de encuentros vivenciales y creativos entre maestros, maestras y otros agentes educativos.

- Se realizaron 3 talleres de imaginarios para activar la relación pedagógica con los espacios del Centro.

- $\quad 15$ Talleres de talleres, los cuales posibilitaron la experimentación con distintas prácticas de formación docente.

- 37 talleres de apropiación para validar y fortalecer la identidad, los principios y las líneas de formación del Centro.

- 24 intervenciones con el Mova Móvil, la estrategia itinerante del Centro, con maestros y maestras de los distintos núcleos educativos de la ciudad.

- 4 encuentros de Mirada Maestra, el ciclo de conferencias diseñado por el equipo de Mova para generar diálogos entre los maestros y las maestras de la ciudad con invitados nacionales e internacionales que se ocupan de diferentes temas educativos.

- 23 encuentros de la agenda satélite de Mirada Maestra, que activaron el intercambio pedagógico y cultural en la ciudad.

- 58 encuentros con diferentes actores para dar cuenta del momento histórico de la propuesta. 
- 9 talleres de cuatro sesiones cada uno, y doce grupos en total, con maestros y maestras de la ciudad para probar algunos de los programas de formación del Centro.

- 24 encuentros en diferentes eventos de ciudad.

- 14 encuentros con comunidades docentes de la ciudad y el país para la construcción de lineamientos de política para la formación de maestros en servicio.

Todas estas estrategias se realizaron en 2014 y 2015 con maestros y maestras de la ciudad, pero sin olvidar los aportes que también podían brindar profesionales provenientes de otras áreas. El principal objetivo que se persiguió con este método de trabajo fue que el diseño de los diferentes aspectos de Mova, tanto espacial como educativo, tuviera un carácter plenamente participativo. De ahí que esta experiencia pueda caracterizarse sin vacilaciones como un ejercicio colectivo desde los saberes de maestros, maestras y otros agentes educativos de Medellín.

\section{Un acontecer en la creación pedagógica}

Los maestros y las maestras de Medellín juegan un papel central en la configuración de Mova. Desde su inicio protagonizaron diálogos constantes, con pares y otros profesionales, que pusieron en escena su acervo académico y pedagógico y la reflexión sobre su práctica. Dicho ejercicio desplaza la tensión que ve al maestro como un agente subordinado a las producciones de los expertos que no le dan más que el papel de reproductor de las teorías a aplicar en la escuela. En el caso de Mova, la relación fue distinta. Los llamados expertos o asesores, y los profesionales, no escribieron algo así como un manual para la construcción de Mova. Por el contrario, el hilo conductor de la conversación se tejió con la voz de los maestros y las maestras de la ciudad, una voz que traía consigo su experiencia, su producción de saber pedagógico, la lectura de la escuela y su contexto y la configuración de otros saberes.

En este sentido, la pedagogía se asumió como un saber alterable, que se reinventa constantemente, que nunca acaba, que se resiste a estar subordinada y atrapada en la uniformidad. $\mathrm{Su}$ existencia se hace evidente en las formas de la práctica pedagógica, que no se convierte en norma, sino en posibilidad de diálogo, interacción y creación de los maestros y las maestras. En palabras de María Silvia Serra (2010, p. 79):

El saber de la pedagogía podría pensarse entonces como un saber que altera — que transforma a los objetos de los que habla-, pero a la vez como un saber parcial o incompleto, en el sentido de unas instrucciones insuficientes y hasta obsoletas en relación a sus efectos.

Desde esta perspectiva, la pedagogía se resiste a modelos, a esquemas fijos y estables. La pedagogía no trata a los objetos positivamente, sino como objetos de saber que se constituyen. Puede afirmarse, entonces, que en Mova se produjo un acontecimiento de creación pedagógica.

\section{Diseño de la propuesta educativa de Mova}

\section{Descripción de la propuesta educativa}

La propuesta educativa de Mova tiene tres componentes: diseño de espacios, contenidos educativos y gestión de comunidades. Estos componentes están íntimamente relacionados entre sí, pero con cada uno se persiguió un propósito específico.

La inclusión del diseño de espacios en esta propuesta apuntó a la reflexión sobre el espacio como un aspecto central de los procesos educativos. Mova no se pensó simplemente como un edificio donde sucederían experiencias educativas, sino que el espacio mismo debía ser un elemento fundamental en la promoción y la configuración de estas experiencias; se concibió, por tanto, como un elemento que debía permitir la conexión de comunidades de maestros y maestras, y propiciar interacciones de educación no formal e informal entre ellos.

Los contenidos educativos se definieron con base en cuatro líneas de acción (desarrollo humano, formación situada, reflexión metodológica e investigación educativa), las cuales generaron una serie de estrategias que se tradujeron en el diseño de programas de formación asociados con cada línea. Estos programas se configuraron con un carácter modular, de modo que maestros y maestras pudieran decidir acerca de sus propias rutas formativas, de acuerdo con sus intereses particulares en módu- 
los pertenecientes a diferentes programas. Los programas juegan el papel de la estructura educativa que contiene y articula cada módulo.

Finalmente, la gestión de comunidades se entendió como la mejor oportunidad para que Mova se convirtiera, no solo en un centro para la formación, sino en un hito cultural de Medellín. Este componente dio a la propuesta educativa un carácter mucho más amplio y ambicioso de lo que podría esperarse en un principio, pues permitió que aunque el motor de Mova fuera el trabajo con los maestros y las maestras, al mismo tiempo pudiera extenderse a otros agentes y públicos de interés, cuya relación con las comunidades pedagógicas fuera beneficiosa.

\section{Maestros y maestras en la tarea de diseñar sus espacios de formación}

Los talleres de imaginarios fueron desarrollados, con docentes de las instituciones educativas públicas y privadas de Medellín, a partir de herramientas generativas o creativas utilizadas por el equipo de diseño de Mova como una estrategia para conectar el discurso del deseo con los espacios educativos; esto exigió re-significar el lugar de maestros y maestras a partir de la cocreación con sus pares y otros profesionales. Dicha situación obligó a revisar el pasado para comprender el presente e imaginar el futuro del Centro, todo a partir de la creación de artefactos que permitieran la remembranza y la proyección desde la sensibilidad del ser maestro o maestra.

Las respuestas dadas a preguntas como: ¿Cómo le gustaría, cómo se sueña, cómo se imagina ese espacio de formación docente?, no se redujeron simplemente a la articulación de una idea, sino que aparecieron como el contenido que devela maneras de vivir la escuela, los contextos y las realidades de la pedagogía, las cuales dan cuenta de los sueños, angustias, proyectos, temores, intereses y necesidades sentidas por las comunidades de docentes.

Hacer estas lecturas de manera reflexiva, desde los diferentes saberes profesionales que entrama Mova, requirió centrar la escucha en la voz de maestros y maestras, entendiéndola como la posibilidad de conocer la subjetividad de otros y otras. Siguiendo a Lenkersdorf (2008), diríamos que esta reflexión hizo énfasis sobre la palabra escuchada, antes que en la palabra hablada, lo cual precisó atender en perspectiva de la otra cultura, del otro contexto, de la otra dimensión del ser, de su identidad, con em- patía y respeto, para lograr así afinar nuestra propia cosmoaudición. En definitiva, abrirse a escuchar el mundo de los demás.

De esta manera, esas enunciaciones transitaron por un proceso de abstracción que permitió develar el sentido de la expresión. Se encontró, por ejemplo, que los espacios iluminados, tan anhelados por los grupos de docentes, se asocian con un estado de tranquilidad. Así que imaginar, preconcebir y prefigurar el espacio se suscribe al propósito de comprender ese vasto universo pedagógico de maestros y maestras, que está lleno de narrativas que dan cuenta de su práctica. Ésta incluye personajes, espacios, anécdotas y reflexiones poderosamente reveladoras, que llaman la atención sobre los mínimos innegociables que debe tener un centro de formación como Mova.

El diseño de los espacios de formación de Mova viajó por el imaginario colectivo de la comunidad docente de la ciudad, y tuvo como premisa pedagógica el espacio como "dispositivo didáctico", en tanto que el espacio se concibió en sí mismo como un detonante de experiencias y sensaciones que genera y dispone aprendizajes pertinentes en relación con los objetivos de formación.

\section{Participación de maestros y maestras en el diseño de contenidos, un desafío pedagógico}

Los contenidos educativos de Mova se delinearon a partir de las siguientes construcciones: la aproximación teórica frente a lo pedagógico para los procesos formativos del Centro; la concepción que guiaría las reflexiones sobre innovación educativa y la decisión sobre las líneas de acción, las estrategias formativas y los programas de formación que tomarían lugar en Mova.

La participación de los maestros en el diseño de estos contenidos tomó varias formas. En primer lugar, se designaron maestros y maestras en comisión, los cuales participaron en diferentes reuniones con el equipo de profesionales de Mova y con integrantes de la Escuela del maestro, para acordar cómo abordar el asunto de la pedagogía. Esto permitió dar definiciones mucho más precisas sobre diferentes elementos de la propuesta educativa, descartar algunos por inapropiados en términos teóricos y conceptuales e incluir otros que darían mayor fortaleza a lo que quería hacerse desde el Centro.

Los maestros en comisión también ayudaron a conceptualizar la perspectiva de formación que finalmente se adoptó, y se encargaron de contribuir a la determinación de las líneas de ac- 
ción de la propuesta educativa y de los contenidos que debían abordarse en cada una de estas líneas. Así mismo, cabe destacar su participación directa en el diseño de los programas de formación que se implementan en cada línea; es decir, desde su conocimiento sobre la profesión docente, para garantizar su formación continuada de acuerdo a las necesidades encontradas en las voces de sus colegas.

Pero la participación de los docentes no se redujo a su cooperación como maestros en comisión. Desde el comienzo, un factor importante para Mova fue la construcción participativa de los distintos componentes del Centro. Esto se consiguió de un modo que puede calificarse como mixto. No se trató de imponer ciertos contenidos educativos a la comunidad de maestros desde un grupo de expertos, pero tampoco se construyeron estos contenidos directamente desde los propios maestros y solo entre maestros. Se buscó más bien una aproximación intermedia. Se realizaban propuestas desde el equipo de maestros y profesionales de Mova y luego se sometían a consideración y al debate en las comunidades de maestros. A partir de ese diálogo, se replanteaban y rediseñaban las propuestas, hasta que se lograba una versión que hubiera sido avalada por un amplio número de sectores.

Así se logró un grado importante de participación de maestros y maestras, pero siempre en diálogo con otros profesionales. Todo, desde la idea de que aunque los maestros son los actores principales en todos los procesos de Mova, al mismo tiempo cabe aceptar que su labor se beneficia enormemente del intercambio de ideas con profesionales provenientes de otros campos del saber. Mova puede describirse por ello como un centro de maestros para maestros, pero donde los aportes de otros se toman en cuenta y se someten a consideración. A continuación se presenta una descripción de la identidad de Mova y de cada una de las líneas de acción como producto de estas largas conversaciones del equipo de profesionales de contenidos de Mova con los maestros y maestras de la ciudad.

\section{Identidad de Mova}

Se definieron tres principios de corte más filosófico que podían servir como antecedente y sustento de los contenidos educativos de Mova.

El primer principio, "ser para dialogar", se relaciona con la socialización y el intercambio de ideas y experiencias. Para este fin se recurre a ambientes de aprendizaje, que si bien fomentan procesos de formación planificados, también entienden la conversación informal como un escenario privilegiado para la construcción e intercambio de saberes. La plática argumentada se posiciona como estrategia clave para la formación de ciudadanías reflexivas, críticas y propositivas con la realidad. El principio "ser para dialogar" conduce así a una concepción del conocimiento que integra una pluralidad de pensamientos, lenguajes y puntos de vista, y propicia nuevas oportunidades para la formación profesional, el esparcimiento y la afectividad. Ser para dialogar lleva entonces a comprender que la subjetividad de maestros y maestras, como la de cualquier otro ser humano, se forma, confronta y expande gracias a su interacción permanente con otros y otras mediante el diálogo.

El segundo principio, "saber para crear", busca establecer canales para que el conocimiento se convierta en una experiencia educativa significativa, en la que maestros y maestras creen dinámicas de trabajo que potencien aprendizajes relevantes y pertinentes. La creación ofrece nuevas opciones para afrontar las problemáticas de aula y, al mismo tiempo, estimula el pensamiento crítico y creativo de las comunidades educativas. Adicionalmente, Mova entiende los procesos creativos como oportunidades para explorar y construir desde intereses estéticos e intelectuales, facilitando ambientes de encuentro propicios para el ejercicio artístico y lúdico. Saber para crear apela, en definitiva, a un tipo de formación que evita la simple memorización de contenidos desarticulados del contexto, y se concentra más bien en la apropiación crítica del conocimiento para ponerlo en práctica bajo los retos que impone cada realidad social particular.

Finalmente, el principio de "crear para innovar" se basa en comprender la formulación de preguntas, la indagación y la investigación como procesos que, si bien se originan en situaciones específicas, pueden llegar a movilizar transformaciones significativas en otros contextos. En este sentido, crear para innovar incluye la reconsideración de prácticas pedagógicas en función de nuevas problemáticas, la renovación de discursos y saberes de acuerdo con las demandas del contexto y el continuo ejercicio docente como motivador de acciones transformadoras. En este punto, el maestro participa de una experiencia que integra su ser, su saber y su crear como punto de partida para la participación en ambientes que promueven acercamientos novedosos a los procesos de educación. 


\section{Líneas de acción de Mova}

Las líneas de acción se plantearon como los medios para alcanzar la realización de todos aquellos elementos explícitos e implícitos en los principios de identidad de Mova.

Línea de desarrollo humano: el bienestar del maestro y la maestra en función de su vocación

Esta línea se fundamenta en la experiencia personal y el diálogo como premisas para la interacción, la comunicación, la investigación y la construcción conjunta de ciudadanías. En este sentido, se sustenta en un trabajo de pares que reconoce la importancia de incentivar la vocación y el bienestar general docente. El propósito es lograr que las capacidades y habilidades inherentes al sujeto se conviertan en un soporte sólido para el fortalecimiento y el desarrollo de sus competencias, tanto en sus aspectos intelectuales y profesionales, como en sus aspectos afectivos y de reconocimiento y valoración social. Se procura, entonces, la reconstrucción de lo humano desde un sentido integral que permita comprender a maestros y maestras como actores y sujetos de reconfiguraciones continuas y en pleno desarrollo de sus habilidades, capacidades y competencias, donde su vocación sea un vínculo consigo mismo y su entorno.

\section{Línea de formación situada: el conocimiento bajo las pre- guntas del contexto}

La formación situada se funda en la reflexión, el análisis y la intención de interactuar con el contexto desde una comprensión de las condiciones sociales y culturales de los maestros y las maestras, de la comunidad educativa y la escuela. Desde esta perspectiva, se entiende como un proceso continuo y dinámico de cualificación que comprende y aplica diferentes saberes en atención a la transformación educativa de ambientes, situaciones y experiencias de la realidad social y cultural de la ciudad y sus habitantes. Así mismo, se busca que la línea permita la comprensión de la realidad social como consideración importante dentro del acto educativo, que concibe el contexto de aula, escuela y ciudad como una experiencia dinámica que nutre a maestros $\mathrm{y}$ maestras de nuevas alternativas con responsabilidad política y ética.

\section{La reflexión metodológica: la pedagogía como cooperación}

En Mova se cree que las metodologías empleadas, por un maestro o una maestra para generar experiencias educativas con sus estudiantes, deben verse como un proceso en constante transformación, que surge del intercambio de ideas con otros y otras. Por ello, la reflexión metodológica se entiende como el diálogo que se establece entre maestros y maestras y otros actores educativos acerca de la formulación, experimentación, diseño, evaluación y sistematización de prácticas y metodologías que potencien procesos educativos en el contexto escolar. Esta línea se aborda desde la perspectiva del pluralismo metodológico, que propone que todo problema práctico o teórico requiere de una reflexión permanente sobre los procedimientos más adecuados para resolverlo, pues cada una de estas situaciones suscita sus propios requerimientos metodológicos. Con ello, la reflexión metodológica se ve en Mova como la posibilidad de que maestros y maestras intercambien experiencias acerca del modo en que sus propias prácticas pueden recontextualizarse en diferentes escenarios educativos, de acuerdo con las particularidades de cada situación.

Investigación educativa: una aproximación crítica a las experiencias educativas

Esta línea se enfoca en la producción de saber pedagógico, disciplinar y científico, desde la propia mirada de maestros y maestras a partir de la investigación, sistematización y socialización de sus prácticas en conversación con teorías pedagógicas y experiencias educativas. La investigación en Mova se pregunta por el contexto, problematiza la práctica, permite que maestros y maestras se vean a sí mismos como sujetos reflexivos, críticos y transformadores de su quehacer. En concordancia con lo anterior, en Mova se enfatizan tres enfoques de investigación educativa: la investigación en educación y pedagogía, la investigación escolar o formativa y la sistematización de experiencias educativas y prácticas pedagógicas.

\section{El tema de la innovación educativa}

Mova se diseñó de tal modo que permitiera la confluencia de múltiples orientaciones y corrientes pedagógicas. Éstas nacen de los diferentes saberes y experiencias personales de los maestros 
y las maestras, y facilitan la formulación de múltiples aproximaciones a la educación dentro y fuera de la escuela. El centro se vale de la innovación educativa como una manera de articular esta pluralidad de visiones sobre la educación y de ayudar a materializarlas de acuerdo con los requerimientos impuestos por cada circunstancia particular. La propuesta de Mova es aproximarse a la noción de innovación educativa desde tres variables que pueden identificarse con los verbos contextualizar, transformar y permear. En este sentido, desde Mova se afirma que hay innovación educativa cuando una práctica pedagógica logra satisfacer tres criterios:

- Es una práctica situada en el contexto de un grupo particular. La práctica se relaciona con problemáticas que afectan directa o indirectamente a las personas de ese grupo y al grupo en conjunto.

- Es una práctica que transforma las experiencias educativas del grupo. Esto significa que la práctica, además de estar contextualizada, tiene una incidencia claramente identificable en los procesos educativos.

- Es una práctica que permea las experiencias educativas de otros grupos. Una práctica contextualizada y transformadora, respecto a un grupo particular, permite también un uso contextualizado que transforma las experiencias educativas de otros grupos que no constituían el foco inicial de la práctica. En este sentido, la práctica alcanza a otros grupos y permea sus experiencias educativas.

Por tanto, una de las finalidades de Mova es promover procesos educativos innovadores en la ciudad, e incluso en el país, a partir de la propia experiencia de los maestros y las maestras. Solo en la medida en que las experiencias significativas de los maestros logren situarse en un contexto, transformar las experiencias educativas de los estudiantes de ese contexto y permear otros contextos, puede empezar a hablarse de que esas experiencias significativas son innovadoras. Y uno de los propósitos es facilitar este desarrollo innovador.

Educación y Cludad No 29 Julio - Diciembre de 2015 ISSN 0123-0425 - PP 80 - 88
Participación de maestros y maestras en la gestión de comunidades

Los maestros y las maestras son dinamizadores en la comunidad educativa y pieza estratégica para el desarrollo social. Ellos articulan a sus estudiantes y sus familias a procesos educativos que se expanden y trascienden hacia otros escenarios sociales. Para restablecer este vínculo social en Mova se diseñaron estrategias, encuentros y reflexiones donde el maestro y la maestra se ponen en escena para dialogar con la ciudad por medio de la divulgación de experiencias entre ellos y otros agentes educativos.

En este sentido, Mova no solo es un centro de formación, sino un espacio que funciona como eje articulador entre la escuela, su contexto y distintos escenarios de la ciudad. Como punto inicial se establecieron diálogos con maestros y maestras de la ciudad y el público en general por medio de diferentes estrategias. Por ejemplo, se activó la divulgación de lo realizado en Mova mediante redes sociales, espacios Web y la instalación en diferentes eventos de la ciudad, para dar a conocer lo relacionado con Mova y atender las preguntas de profesionales y otros públicos.

También se realizaron recorridos por la ciudad para encontrar, junto a maestros y maestras, el valor educativo de diversos espacios además del de la escuela y los centros tradicionales de formación. El recorrido se realizaba en bus, caminando y apoyados en el recuerdo, la palabra y la descripción, para luego usarlos como objetos de enseñanza. Otras estrategias como Mirada Maestra y la participación en distintos eventos de la ciudad, permitieron el desplazamiento de diferentes invitados nacionales e internacionales a las instituciones educativas para dialogar sobre temas educativos específicos. Estos fueron encuentros abiertos, no solo a la comunidad pedagógica, sino a otros profesionales y la comunidad en general.

Todas estas estrategias se articularon mediante un plan de comunicaciones y apropiación, liderado por el equipo de Mova, pero alimentado por la respuesta de los distintos públicos a lo que podría resultar más o menos eficaz respecto a los diferentes tipos de relación que podría mantener Mova, no solo con maestros y maestras, sino con otros agentes educativos. La gestión de comunidades ha buscado convertir a Mova en un hito cultural de la ciudad que permita a otros públicos llegar al Centro para el intercambio de saberes con comunidades de estudiantes, intelectuales, grupos de investigación, familias y otras instituciones como museos, bibliotecas, radio y televisión, entre otras. 
El propósito que se ha ido logrando poco a poco es que la gestión de comunidades ayude a establecer nuevos imaginarios sobre los maestros y maestras por medio de la divulgación y visibilización de sus prácticas, además, se ha conseguido fomentar la creación de redes de interaprendizaje y clubes que den sentido a una comunidad docente activa, preocupada por su formación y por la importancia de ésta en el mejoramiento de la calidad del sistema educativo en general.

\section{A modo de cierre}

Mova se enfoca en la formación de los maestros y las maestras en servicio, de Medellín, desde una propuesta educativa heterogénea e incluyente que fomenta el diálogo y el intercambio de ideas sobre saberes y prácticas pedagógicas. Es una propuesta educativa que se orienta hacia fortalecer e impulsar procesos de pensamiento de diferente índole, como el pedagógico y el tecnológico, el analítico y el emocional, el estético y el corporal, entre otros. Además, Mova busca fortalecer saberes y contenidos asociados con cada uno de estos procesos. Privilegia y busca la formación de maestros reflexivos de sus prácticas pedagógicas, en diálogo con las redes académicas de distintas disciplinas por medio de su producción de saber pedagógico.

La innovación educativa se convierte aquí en eje transversal de los procesos formativos. Se busca con ella que el Centro motive al maestro a pensar el aula como un escenario de problematización de saberes que pueden re-contextualizarse para permear y transformar una realidad educativa particular. La apuesta por la formación pedagógica como proyecto colectivo de ciudad, dio lugar a pensar en la continuidad del sentido de Mova desde un fundamento polisémico, donde concurran voces en la búsqueda de caminos alternativos y movilizadores de formación. Es aquí donde se instala el interés por la adopción de una política pública de formación de maestros y maestras en servicio, pensando en que la sostenibilidad de este proyecto debía ir más allá de un período de gobierno. Con ello se busca que la formación docente tenga un direccionamiento de largo aliento, coherente con la realidad de la ciudad.

Esto sugirió abordar la construcción de unos lineamientos que dieran un horizonte a la formulación de esa política de formación docente, desde tres miradas: una, que va hacia el direccionamiento estratégico de las perspectivas de ciudad en clave de innovación educativa; otra, en relación con el escenario actual de formación de la ciudad, de cara a los referentes nacionales e internacionales, y la tercera, hacia las voces de los diferentes actores educativos que constituyen una amalgama de intereses, necesidades y expectativas desde sus diferentes posturas, pensamientos y momentos históricos en la ciudad. Todo esto con miras a la perduración en el tiempo de uno de los propósitos principales de Mova: contribuir al diálogo de saberes entre maestros y maestras con otros agentes educativos, para pensar de un modo mucho más amplio y ambicioso la formación en servicio de nuestros docentes. 


\section{Referencias}

Alcaldía de Medellín. (2012). Plan de Desarrollo de Medellín. Medellín, un hogar para la vida. 2012-2015. Medellín.

Secretaría de Educación de Medellín. (2014). Ruta para el mejoramiento de la calidad de la educación: Líneas rectoras. Medellín.

Secretaría de Educación de Medellín, Parque Explora, Proantioquia. (2014). Mova, Centro de Innovación del maestro: Propuesta Educativa. Medellín. En proceso de publicación.
Serra, M. S. (2010) ¿Cuánto es una pizca de sal? Acerca del juego de la transmisión y las reglas de la pedagogía. En Frigerio, G., y Diker, G. Educar: saberes alterados. Buenos Aires: Del Estante Editorial, p.10.

Lenkersdorf, C. (2008). Aprender a escuchar. México: Plaza y Valdés. 